\title{
Effect of Freeze-Thawing Process on the Size and Lamellarity of PEG-Lipid Liposomes
}

\author{
Supaporn Sriwongsitanont and Masaharu Ueno*
}

Graduate School of Pharmacy, University of Toyama, 2630 Sugitani, Toyama, 930-0194 Japan

\begin{abstract}
The influence of the freeze-thawing process on the size and lamellarity of multilamellar PEG-lipid liposomes prepared from a mixture of egg yolk phosphatidilcholine (EggPC) and distearoyl phosphatidylethanolamine polyethyleneglycol (DSPE-PEG) 2000 was investigated. Trapped volume measurement, quasielastic light scattering (QELS) and freeze-fracture electron microscopy were used to estimate the morphology and lamellarity of liposomes. During the freeze-thawing process, the lamellarity of multilamellar vesicles (MLVs) depended strongly on both PEG-lipid concentration and the number of freeze-thaw cycles. The decrease in the number of lamellae was a function of the number of freeze-thaw cycles. The increase in trapped volume coincided with the decrease in the number of lamellae observed in electron micrographs. After comparing the results obtained from EggPC/DSPE-PEG2000 MLV and from pure EggPC MLV, it was concluded that during the freeze-thawing the liposomes with DSPE-PEG2000 achieved a unilamellar structure more readily than the pure EggPC liposomes.
\end{abstract}

Keywords: freeze-thaw; lamellarity; trapped volume; liposome; PEG-lipid; freeze-fracture electron microscopy.

\section{INTRODUCTION}

Multilamellar vesicles (MLVs) are spontaneously formed when phospholipid molecules are dispersed in an excess volume of aqueous solution. MLVs are quite heterogeneous both in size and in number of lamellae. Since the structure of MLV consists of concentric bilayers separated by narrow aqueous spaces, the encapsulation capacity of MLVs is very low. Larger trapped volumes can be achieved by decreasing lamellarity using a variety of procedures including freezethaw, dehydration-rehydration, and reverse phase [1].

Freezing and thawing method is convenient for increasing the trapped volume of MLV preparations [2-4]. The mechanism by which the freeze-thawing process increases the trapped volume is not clear. However, it likely reflects a physical disruption of lamellar structure, probably due to ice crystals formed during the freezing process $[1,5]$. While the influences of the freeze-thawing process on the physical properties of the lipid bilayer have been described in many reports [1-8], few studies have attempted to investigate the effect of freeze-thawing on the formation of liposomes containing polyethylene glycol (PEG)-lipid [9-11]. The presence of hydrophilic PEG molecules on the surface of the bilayer could affect vesicle structure change during the freezethawing process. In a previous study, we have reported the effect of freeze-thawing on the size and size distribution of PEG-lipid liposomes prepared with the detergent removal method [9].

In the present study, we investigated the freeze-thawing effects on the size and lamellarity of multilamellar liposomes

\footnotetext{
*Address correspondence to this author at the Graduate School of Pharmacy, University of Toyama, 2630 Sugitani, Toyama, 930-0194 Japan; Tel: +81-76-434-7565; Fax: +81-76-434-5050;

Email: mueno@pha.u-toyama.ac.jp
}

constituted of EggPC/DSPE-PEG2000 prepared by Bangham method [12]. The mean diameter was measured using the light scattering method and the trapped volume of each freeze-thaw cycle was determined by fluorometric method. The morphological change and lamellarity of vesicles were also illustrated by freeze-fracture electron microscopy. The ${ }^{31} \mathrm{P}-\mathrm{NMR}$ signal intensity measurement, which is a straightforward procedure for determining the lamellarity of phospholipid dispersion, has been frequently used $[1,6,13]$. Although freeze-fracture electron microscopy cannot provide the accurate number of lamellae, this method is useful for observing the morphological structure of the liposomes and internal lamellar structure. To examine how the inclusion of PEG-lipid affects the alteration of size and lamellarity of liposomes upon the process of freeze-thawing, a measurement on EggPC MLVs without DSPE-PEG2000 was also conducted.

\section{MATERIALS AND METHODS}

Egg yolk phosphatidylcholine (EggPC; purity of $\mathrm{PC}=98.8 \%)$ and N-methoxy polyethyleneglycolsuccinyldistearoyl phosphatidylethanolamine (DSPE-PEG2000; 98\%) were purchased from Nihon Yushi (Tokyo, Japan). All other chemicals were analytical grade and used without further purification.

\subsection{Preparation of Liposomes}

Multilamellar liposomes were prepared by the hydration method (Bangham method) [12]. EggPC and DSPEPEG2000 were dissolved together in $\mathrm{CHCl}_{3}: \mathrm{CH}_{3} \mathrm{OH} 1: 2$ (v/v) mixture. The mixture was dried to a thin film under a stream of nitrogen. Then the lipid film was stored under vacuum more than $6 \mathrm{~h}$ to remove the traces of organic solvent. The dry lipid film was hydrated by the addition of 
$20 \mathrm{mM}$ Tris buffer with $150 \mathrm{mM} \mathrm{NaCl}$ and then vigorously shaken with a vortex mixer (vortexing) for 1 min to produce MLVs.

The MLV suspensions were frozen and thawed for one to ten cycles. A single freeze-thaw cycle consisted of freezing for $5 \mathrm{~min}$ at liquid nitrogen temperature $\left(-196^{\circ} \mathrm{C}\right)$ and thawing for $5 \mathrm{~min}$ in a water bath at $40^{\circ} \mathrm{C}$.

\subsection{Size Measurements}

The average diameters of vesicles were measured using a quasielastic light scattering apparatus (Photal ELS-6000, Otsuka Electronics, Osaka, Japan) at $25^{\circ} \mathrm{C}$.

\subsection{Determination of Trapped Volume}

Trapped volumes of liposomes were determined according to method of Oku et al. [14] with slight modification as follows. $100 \mu \mathrm{l}$ of liposome suspension, which was prepared in $10 \mu \mathrm{M}$ of a calcein solution, was diluted by $2.5 \mathrm{ml}$ of 20 $\mathrm{mM}$ Tris buffer containing $150 \mathrm{mM} \mathrm{NaCl}$. The fluorescence of external calcein was quenched by adding $60 \mu \mathrm{l}$ of 100 $\mathrm{mM} \mathrm{CoCl}$. The fluorescence intensity of the sample was measured before and after addition of $\mathrm{CoCl}_{2}$ on a fluorescence spectrometer (Shimadzu, RF-500, Tokyo, Japan) (excitation $\lambda=490 \mathrm{~nm}$, emission $\lambda=520 \mathrm{~nm}$ ). Then $20 \mu \mathrm{l}$ of $10 \%$ Triton X-100 was added to release the entrapped calcein and fluorescence intensity was measured again to determine the background fluorescence intensity. The entrapment efficiency was calculated by the following equation:

Trapped Volume $(\%$ of Total $)=\left(f_{\text {in }} \cdot r_{1}-f_{\text {total.q }} \cdot r_{2}\right) /\left(f_{\text {total }}-f\right.$ total.q $\left.r_{2}\right) \times 100$

where $f_{\text {in }}$ is the fluorescence intensity of the internal calcein; $f_{\text {total }}$ and $f_{\text {total. } q}$ are the total fluorescence intensity of calcein before and after vesicle destruction, respectively; $r_{1}$ and $r_{2}$ are correction factors for the volume increase upon adding a $\mathrm{CoCl}_{2}$ solution and Triton X-100 solution, respectively. They were $2660 / 2600$ and $2685 / 2600$ in our experiments. In the experiments, it is assumed that calcein is equally distributed between the vesicles interior and the bulk medium.

In combination with information on size, the trapped volume can be used as an indicator of vesicle lamellarity. Therefore, to investigate further how the presence of PEG-lipid and freeze-thawing process affect the number of lamellae of liposomes, we compared the determined trapped volume (experimental value) with the internal volume of the vesicles calculated from the mean diameter of the vesicles (theoretical value) and expressed it as ratio of trapped volume. The Ratio of trapped volume was calculated according to the following equation:

\section{Ratio of Trapped Volume $=$ Trapped Volume $($ exp) $/$ Trapped Volume $_{(c a l)}$}

Where trapped volume (exp) is the calcein trapped volume of liposomes obtained by direct experiments, and trapped volume $($ cal $)$ is the theoretical value of trapped volume of the vesicles calculated from particle size of unilamellar liposomes assuming an area per molecule of $0.7 \mathrm{~nm}^{2}$ and a bilayer thickness of $4 \mathrm{~nm}$ [15]. The change in the ratio of trapped volume will reflect the change in the number of bilayer per vesicle. If the trapped volume from experiment is substantially less than the theoretical value of unilamellar vesicles with the same size, then appreciable proportion of the vesicles must be multillamellar.

\subsection{Freeze-Fracture Electron Microscopy}

Liposome preparations were mixed with $10 \%$ (v/v) glycerol solution and then rapidly frozen in liquid nitrogen (196 ). The samples were fractured in a freeze replica apparatus (FR-7000B, Hitachi, Japan) at -120 . Immediately after fracturing the sample, an electric discharge was used to coat the fracture faces with $\mathrm{Pt} / \mathrm{C}$ followed by $\mathrm{C}$ at an angle of $45^{\circ}$ and $90^{\circ}$ for each coating, respectively. The replicas were stripped off from holder and cleaned by submersion in solution of commercial bleaches and distilled water. The cleaned replicas were mounted on 300-mesh Ni grids, dried, and examined in a transmission electron microscope (JEM200 CX, JEOL, Tokyo, Japan).

\section{RESULTS}

\section{Particle Sizes}

Inclusion of DSPE-PEG2000 into EggPC MLVs resulted in a decrease in the average particle sizes. The average diameter of EggPC MLVs decreased from $4 \mu \mathrm{m}$ to less than 1 $\mu \mathrm{m}$ when DSPE-PEG2000 was incorporated (Fig. 1). When the MLVs were subjected to freeze-thaw cycles, the average diameters of all preparations, especially EggPC MLVs, decreased with the number of freeze-thaw cycles.

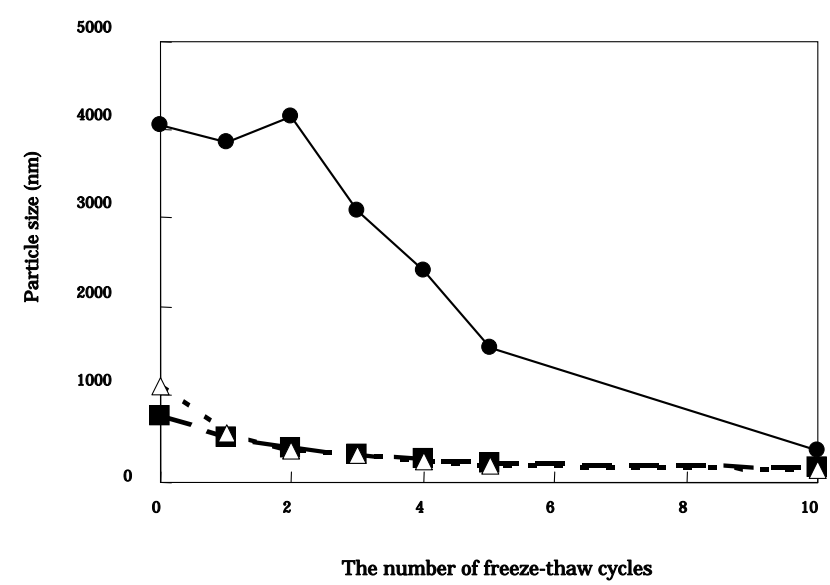

Fig. (1). Influence of the number of freeze-thaw cycle on the mean diameter of MLVs. • EggPC; $\mathbf{\square}$, EggPC/1mol\% DSPE-PEG2000; $\Delta$, EggPC/5mol\% DSPE-PEG 2000.

\section{TRAPPED VOLUME}

The trapped volumes of EggPC MLVs with and without DSPE-PEG2000 are shown in Fig. 2. Before freeze-thawing, in spite of being a significantly smaller size, the trapped volumes of EggPC/DSPE-PEG2000 MLVs were greater than that of EggPC MLVs. When MLVs were subjected to the freeze-thawing process, the trapped volumes of all liposome preparations increased after only one freeze-thawing cycle. Subsequent increases in the number of freeze-thaw cycles of EggPC MLVs resulted with a further increase of the trapped volume, and then the trapped volume began to decrease while freeze-thawing over 4 cycles. In the case of the MLVs of EggPC/DSPE-PEG2000 after being freeze-thawed for 2 cycles, the trapped volume decreased along with the number 


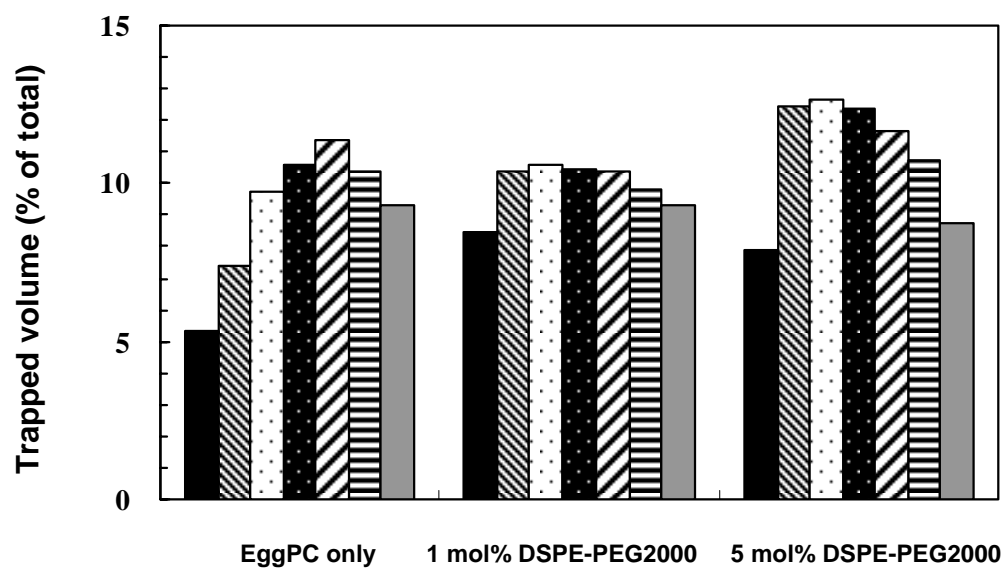

Fig. (2). Calcein trapped volume of EggPC, EggPC/1mol\%DSPE-PEG2000, and EggPC/5mol\%DSPE-PEG2000 as a function of the number

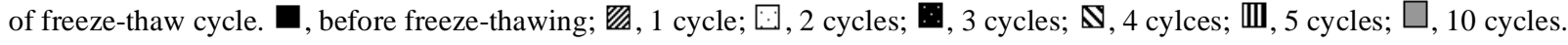

of freeze-thaw cycle. It is important to know that the mean diameters of MLVs decreased along with the number of freeze-thaw cycles as shown in Fig. 1. Theoretically, if liposomes are unilamellar, the larger the sizes gives the higher the trapped volumes. Therefore, to estimate the lamellarity of the vesicles, we compared the trapped volume from the experiment (data in Fig. 2) with the trapped volume calculated from the mean diameter of the same preparation (data in Fig. 1) and expressed it as the ratio of trapped volume. The ratios of the trapped volume of EggPC liposomes and DSPE-PEG2000-containing EggPC liposomes as a function of the number of freeze-thaw cycles are shown in Fig. 3. Before freeze-thawing, the ratio of trapped volume of liposomes containing DSPE-PEG2000 was remarkably higher than that of EggPC MLVs; the ratio of the trapped volume of EggPC before freeze-thawing was 0.04, whereas the ratio of EggPC/DSPE-PEG2000 was higher than 0.2. This higher ratio of trapped volume indicated a structural change towards a less multilamellar structure by containing DSPE-PEG 2000.

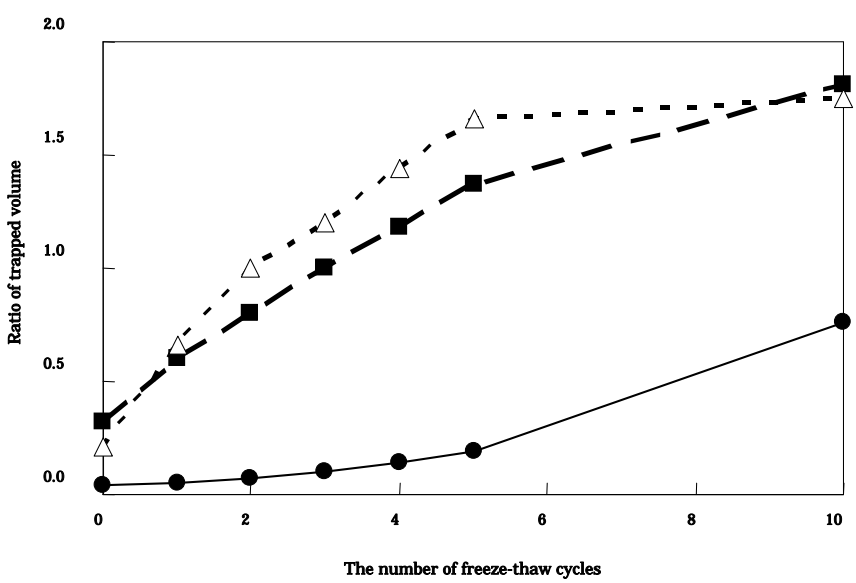

Fig. (3). The ratio of trapped volume of EggPC without DSPEPEG2000 (•),EggPC with $1 \mathrm{~mol} \%$ (ם) and $5 \mathrm{~mol} \%$ ( ) DSPEPEG2000 as a function of the number of freeze-thaw cycle.

After freeze-thawing, all liposome preparations exhibited the ratio of the trapped volume more than those of nonfreeze-thawed liposomes and the ratio of the trapped volume increased with the number of freeze-thaw cycles. It was clearly demonstrated that the increase in the ratio of the trapped volume of EggPC/DSPE-PEG2000 MLVs was significantly higher than that of EggPC MLVs. Furthermore, EggPC MLVs containing 5 mol\%DSPE-PEG2000 exhibited a greater increase in the ratio of trapped volume than EggPC/1 mol\% DSPE-PEG2000.

\section{FREEZE-FRACTURE ELECTRON MICROSCOPY}

Freeze-fracture electron micrographs of EggPC MLVs are shown in Fig. 4. In the absence of DSPE-PEG2000 the liposomes frequently aggregated into large clusters of deformed liposomes (Fig. 4a). Freeze-fracture micrographs, which have fractured cross sections, revealed the close apposition and large number of inner lamellae (Fig. 4 a-f). These tightly stacked inner lamellar vesicles are associated with typical MLVs.

Subjecting MLVs to freeze-thaw cycles resulted with dramatic change in the liposome morphology. The distored structures disappeared. Only spherical vesicles with various sizes were observed (Fig. 5a). Fig. (5 b-f) show freeze fractured micrographs of EggPC MLVs after being freezethawed for 5 cycles. It was clearly illustrated that although the tightly packed concentric bilayers still existed (Fig, 5b), the majority of vesicles had larger interlamellar spaces (Fig. 5 c-d). Moreover, the vesicles within a vesicle (Fig. 5e) and vesicles between the lamellae (Fig. 5 c-d) which were not seen in the sample before the freeze-thawing were observed. Fig. (5f) shows the freeze fractured image of the vesicle which has almost no internal lamellar but does have an inner vesicle.

Incorporation of PEG-lipids into MLVs affected the morphology and lamellarity of the lipid vesicles. The nearly perfectly spherical vesicles were observed when DSPEPEG2000 was incorporated into vesicles. A greater proportion of small vesicles were detected as the PEG-lipid concentration was increased. The freeze-fracture electron micrographs of the EggPC vesicles with DSPE-PEG2000 in the concentration of $1 \mathrm{~mol} \%$ and $5 \mathrm{~mol} \%$ before freeze-thawing are shown in Fig. (6 a-d) and e-h, respectively. A small population of liposomes with dense concentric inner lamellar structure were still present (Fig. 6 a and e), but the interlamellar spaces in EggPC/DSPE-PEG2000 vesicle were obviously larger than those in pure EggPC vesicles. In coexistence with the dense concentric inner lamellar vesicles, vesi- 


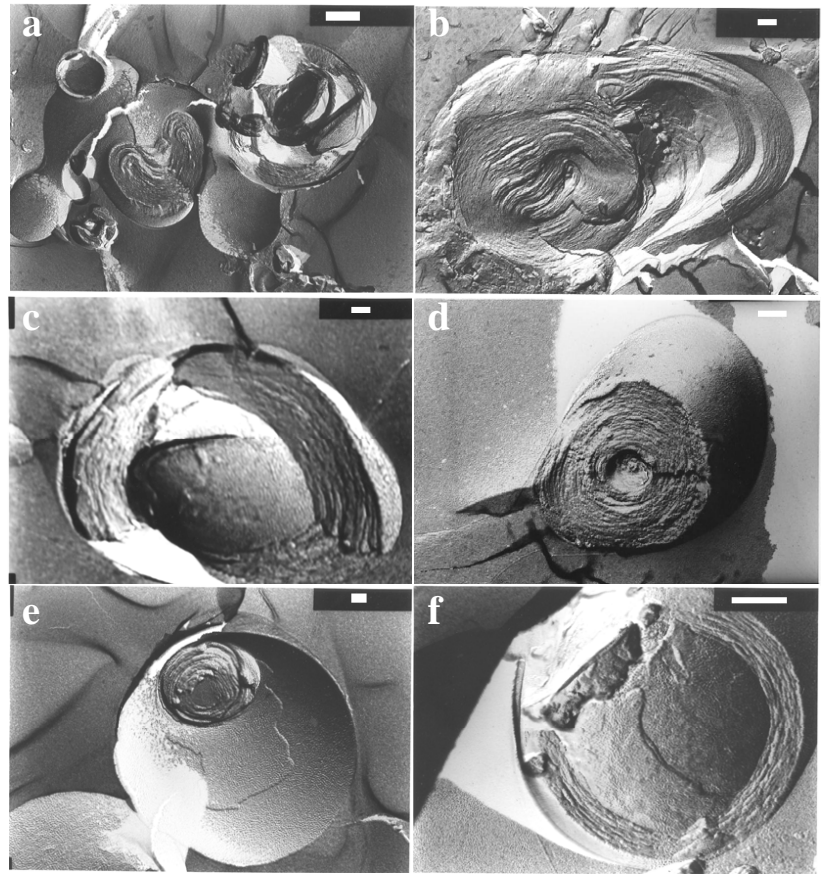

Fig. (4). Freeze-fracture micrographs of EggPC MLVs. Each bar represents $200 \mathrm{~nm}$.

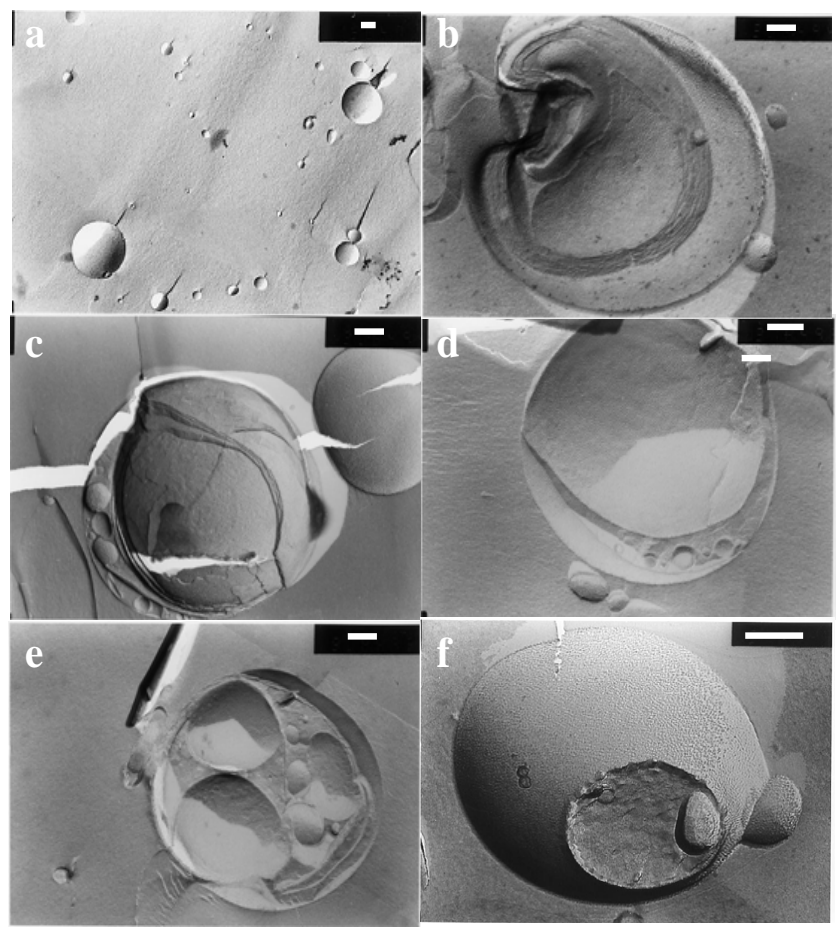

Fig. (5). Freeze-fracture micrographs of EggPC MLVs subjected to five freeze-thaw cycles. Each bar represents $200 \mathrm{~nm}$.

cles with large interlamellar space are frequently observed (Fig. 6 b-c and f-g). The unilamellar structures which were absenct in EggPC MLVs without freeze-thawing were also illustrated (Fig. $\mathbf{6} \mathbf{d}$ and $\mathbf{h}$ ).

The freeze-thawing of liposomes containing DSPEPEG2000 resulted with a very similar sequence of morpho-

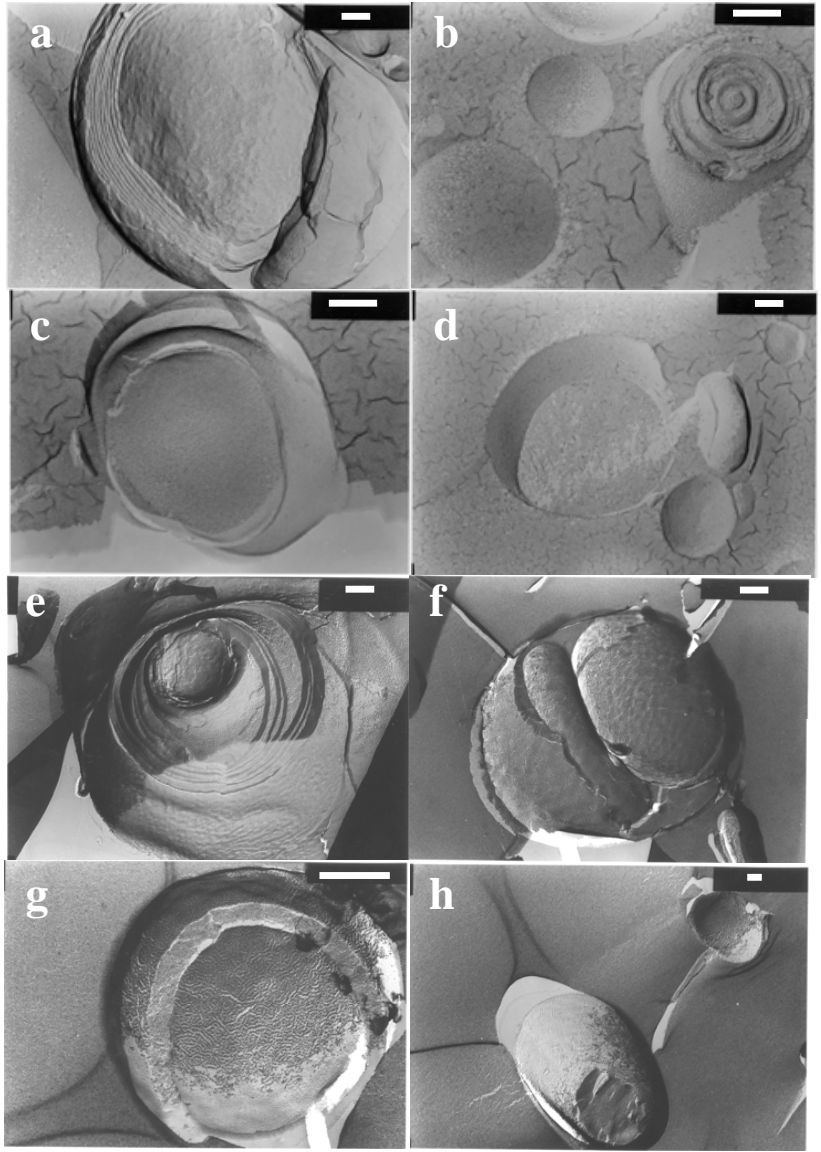

Fig. (6). Freeze-fracture micrographs of EggPC MLVs with 1 mol\% (a-d) and $5 \mathrm{~mol} \%$ (e-h) of DSPE-PEG2000 without freezethawing. Each bar represents $200 \mathrm{~nm}$.

logical transitions as observed in EggPC MLVs. After freeze-thawing, only spherical vesicles with a high polydisperse in size distribution were observed (Fig. 7a). The cross fracture electron micrographs demonstrated that no tightly packed concentric bilayer was found; only vesicles with large spaces between bilayers were observed (Fig. 7 b-f). It is worthwhile to compare the large interlamellar space of the vesicles, obtained in the presence of PEG-lipid after freezethawing, to the tightly packed structure in the absence of PEG-lipid. The distances between the lamellae of EggPC/ DSPE-PEG2000 liposomes were found to be significantly larger than that of EggPC MLVs. Similar to the vesicles without PEG-lipids, freeze-thawing the PEG-lipid liposomes also lead to the formation of small vesicles within a liposome (Fig. 7e) and vesicles in lamellar space (Fig. 7f) but the emerging frequency of the vesicles were significantly smaller than those in the liposomes without the presence of PEG-lipid.

\section{DISCUSSION}

The presence of DSPE-PEG2000 as low as $1 \mathrm{~mol} \%$ in MLVs already affected the size and number of lamellae without freeze-thawing. The decrease in the size of vesicles coincided with the increase in the trapped volume, which clearly proposed that the lamellar of the vesicles decreased when DSPE-PEG2000 was incorporated. It has been demon- 


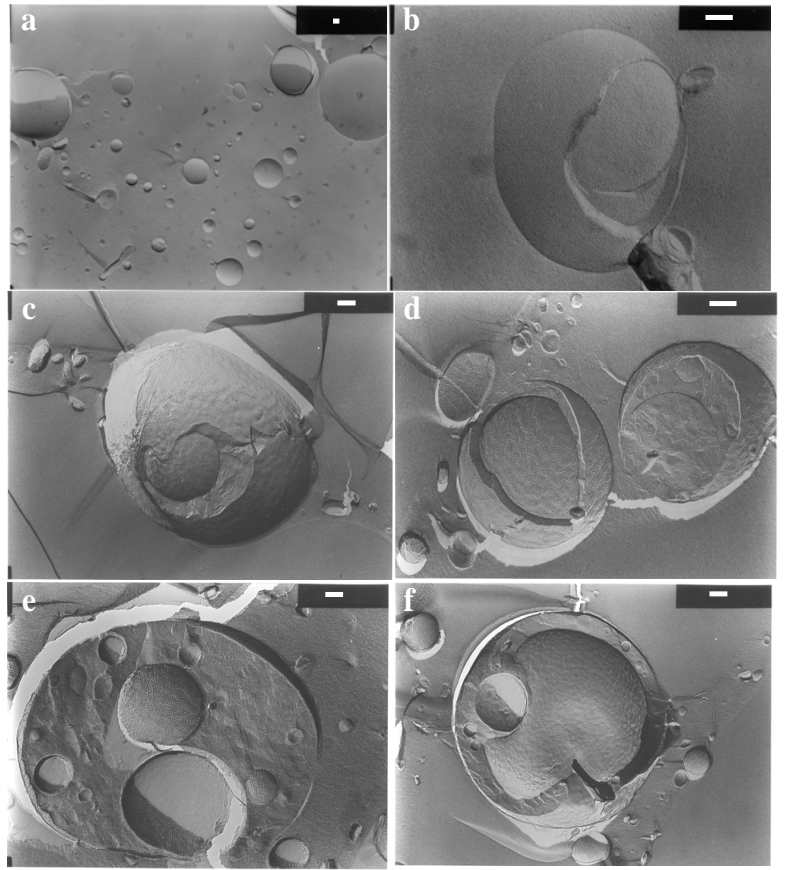

Fig.(7). Freeze-fracture micrographs of EggPC MLVs with $1 \mathrm{~mol} \%$ (a-b) and $5 \mathrm{~mol} \%$ (c-f) of DSPE-PEG2000 after five cycles freezethawing. Each bar represents $200 \mathrm{~nm}$.

strated by the use of the X-ray diffraction method that the distance between bilayer surfaces increases while increasing the PEG-lipid concentration [16-19]. Belsito et al. also demonstrated with spectrophotometric measurements that the addition of PEG-lipids causes a reduction in the number of lamellae [20]. However, the freeze-fracture micrographs in our study provide additional information about the structure of internal lamellae inside the liposomes. The results from the ratio of trapped volume and freeze-fracture electron micrographs clearly showed that the inclusion of PEG-lipid alters the liposomes from multilamellar to oligolamellar liposomes.

After freeze-thawing, the ratio of the trapped volumes of EggPC/DSPE-PEG2000 increased remarkably (Fig. 3) and freeze-fracture micrographs clearly revealed the significant decrease in the number of lamellar (Fig. 7) as well as the absence of the dense concentric inner lamellar. This indicates that almost all of PEG-lipid liposomes are oligolamellar vesicles or unilamellar vesicles. This result is in contrast to the observations made for the freeze-thaw EggPC MLVs where the trapped volume (Fig. 3) was relatively low and the dense concentric lamellar structures were still frequently observed (Fig. 5). It is well known that the freeze-thawing of liposomes results with a dramatic change in trapped volume $[4,6]$. During freezing, the bilayer of the liposomes can be destabilized through expansion of inner water phase by ice formation [8], dehydration of hydrated head group of lipid and so on. During thawing, exposed hydrophobic cores fuse to form new vesicles [21] with decreasing of lamellarity. Our results indicated that the unilamellar formation using the freeze-thawing process of PEG-lipid liposomes is achieved more easily than that of pure EggPC liposomes. A fusogen
PEG [22] could facilitate the fusion event in the process of the freeze-thawing.

We also demonstrated that the decrease in the number of lamellae during the freeze-thawing process also depends on the concentration of PEG-lipids. The rate of increase in the ratio of the trapped volume of 5 mol\%DSPE-PEG2000 is higher than that of $1 \mathrm{~mol} \%$ of DSPE-PEG2000. The acceleration effect of PEG-lipids on the decrease in the number of lamellae after undergoing the freeze-thawing process is not entirely clear. Probably the rapid decrease in the number of bilayers is mainly caused by the presence of strongly hydrated PEG-groups extending from the liposome surface. Several factors, dehydration by PEG, different mode of formation and melting of ice between free water and hydrated water on PEG-group and steric hindrance of PEG-group should cause the unstabiliztion of the lamellar structure, resulting in facilitating the fusion between adjacent lamellae in the process of freeze-thawing, although it is still hard to restrict the factors. The decrease in lamellarity should result in the large internal volume of the liposomes.

Theoretically, if the vesicles are unilamellar, the ratio of experimentally obtained trapped volume and the theoretically calculated trapped volume would be nearly 1 . From our result, in the case of EggPC/DSPE-PEG2000 liposomes (Fig. $3)$ the ratio of the trapped volume after ten cycles of freezethawing was nearly 2 . A possible explanation for this result may be that the theoretical trapped volume was underestimated. In our study we calculated the theoretical trapped volume using the mean diameter obtained by light scattering method, which is the simple and rapid method to analyze. However, the disadvantage of this technique is that the misleading results can be obtained for heterogeneous systems. As mentioned in the Introduction and demonstration of the freeze-fracture electron micrograph (Fig. 4-7), size distribution of the MLVs is heterogeneous; the very large vesicles coexist with the small vesicles. The mean diameter of the freeze-thawed EggPC/DSPE-PEG2000 vesicles determined by the light scattering method was about $200 \mathrm{~nm}$. However in freeze-fracture electron micrograph the very large vesicles with a diameter over than $1 \mu \mathrm{m}$ were frequently observed (Fig. 7 a-c, e-f). Therefore, the measurement of the mean diameter of MLVs based on light scattering lead to the underestimation of the theoretical trapped volume, causing the ratio of trapped volume to be over 1 . Nevertheless, a comparison of the ratio of trapped volume in our experiment can provide the useful information of the lamellarity of the vesicles. It can be assured from the ratio of trapped volume that the number of lamellae of freeze-thawed EggPC/DSPEPEG2000vesicles is appreciable less than that of $\mathrm{EggPC} /$ DSPE-PEG2000 vesicles without freeze-thawing and less than the pure EggPC vesicles with and without freezethawing.

In comparing ${ }^{31} \mathrm{P}-\mathrm{NMR}$ and freeze-fracture electron microscopy, while ${ }^{31} \mathrm{P}-\mathrm{NMR}$ only determines the ratio of the amount of outer to inner phospholipids, freeze-fracture electron microscopy not only shows the number of lamellae, but also reveals the shape, size, and inner lamellar structure of liposomes. Recently, Frohlich et al. demonstrated that the determination of the lamellarity of liposomes is only correct when the lamellae of liposomes are very close together. It is 
not suitable for determining the number of lamellar if the liposomes are oligolamellar where the inner layers have a decreasing surface area [23].

Investigations based on a combination of the light scattering method, trapped volume, and freeze-fracture electron microscopy in our study clearly demonstrated the effect of freeze-thawing on the size and lamellarity of PEG-lipid liposomes. Similar to the MLVs in the absence of PEG-lipid, the size and lamellarity of MLVs containing PEG-lipid decreased during the freeze-thawing process. However, it is worth noting that the inclusion of PEG-lipids further enhanced these increases, indicating that PEG-lipids have influence on the EggPC bilayer during the freeze-thawing procedure. Unilamellar structures are attained more readily during the freeze-thawing of vesicles with PEG-lipids.

\section{ACKNOWLEDGMENT}

This research was supported by a research grant from Japan Society for the Promotion of Science (P 05165).

\section{REFERENCES}

[1] Hope, M.J.; Bally, M.B.; Mayer, L.D.; Janoff, A.S.; Cullis, P.R. Generation of multilamellar and unilamellar phospholipids vesicles. Chem. Phys. Lipids, 1986, 40, 89-107.

[2] Ohsawa, T.; Miura, H.; Harada, K. Evaluation of a new liposome preparation technique, the freeze-thawing method, using Lasparaginase as a model drug. Chem. Pharm. Bull., 1985, 33, 2916-2923.

[3] Ohsawa, T; Miura, H; Harada, K. Improvement of encapsulation efficiency of water-soluble drugs in liposomes formed by the freeze-thawing method. Chem. Pharm. Bul., 1985, 33, 3945-3952.

[4] Mayer, L.D.; Hope, M.J.; Cullis, P.R.; Janoff, A.S. Solute distributions and trapping efficiencies observed in freeze-thawed multilamellar vesicles. Biochim. Biophys. Acta, 1985, 817, 193-196.

[5] Castile, J.D.; Taylor, K.M. Factors affecting the size distribution of liposomes produced by freeze-thaw extrusion. Int. J. Pharm., 1999, $188,87-95$

[6] Hope, M.J.; Bally, M.B.; Webb, G.; Cullis, P.R. Production of large unilamellar vesicles by a rapid extrusion procedure. Characterization of size distribution, trapped volume and ability to maintain a membrane potential. Biochim. Biophys. Acta, 1985, 812, 5565.

[7] Elorza, B.; Elorza, M.A.; Sainz, M.C.; Chantres, J.R. Comparison of particle size and encapsulation parameters of three liposomal preparations. J. Microencapsul., 1993, 10, 237-248.
[8] MacDonald, R.C.; MacDonald, R.I. Applications of freezing and thawing in liposome technology, in: Gregoriadis, G., Ed.: Liposome Technology, CRC Press: Boca Raton, 1984, 1, pp. 209-228.

[9] Sriwongsitanont, S.; Ueno, M. Effect of PEG lipid (DSPEPEG2000) and freeze-thawing process on phospholipids vesicle size and lamellarity. Colloid Polym. Sci., 2004, 282, 753-760.

[10] Sriwongsitanont, S.; Ueno, M. Effect of freeze-thawing and polyethylene glycol (PEG) lipid on fusion and fission of phospholipid vesicles. Chem. Pharm. Bull., 2004, 52, 641-642.

[11] Ueno, M.; Sriwongsitanont, S. Effect of PEG-lipid on fusion and fission of phospholipids vesicles in the process of freeze-thawing. Polymer, 2005, 46, 1257-1267.

[12] Bangham, A.D.; Standish, M.M.; Watkins, J.C. Diffusion of univalent ions across the lamellae of swollen phospholipids. J. Mol. Biol., 1965, 13: 238-252.

[13] Gruber, H.J.; Schindler, H. External surface and lamellarity of lipid vesicles: a practice-oriented set of assay methods. Biochim. Biophys. Acta, 1994, 1189, 212-224.

[14] Oku, N.; Kendall, D.A.; MacDonald, R.C. A simple procedure for the determination of the trapped volume of liposomes. Biochim. Biophys. Acta, 1982, 691, 332-340.

[15] Mimms, L.T.; Zampigi, G.; Nozaki, Y.; Tanford, C.; Reynolds, J. A. Phospholipid vesicle formation and transmembrane protein incorporation using octylglucoside. Biochemistry, 1981, 20, 833-840.

[16] Kenworthy, A.K.; Simon, S.A.; McIntosh, T.J. Structure and phase behavior of lipid suspensions containing phospholipids with covalently attached poly(ethylene glycol). Biophys. J., 1995, 68, 19031920.

[17] Kenworthy, A.K.; Hristova, K.; Needham, D.; McIntosh, T.J. Range and magnitude of the steric pressure between bilayers containing phospholipids with covalently attached poly(ethylene glycol). Biophys. J., 1995, 68: 1921-1936.

[18] Needham, D.; McIntosh, T.J.; Lasic, D.D. Repulsive interactions and mechanical stability of polymer-grafted lipid membranes. Biochim. Biophys. Acta, 1992, 1108, 40-48.

[19] Hristova, K.; Needham, D. Phase behavior of a lipid/polymer-lipid mixture in aqueous medium. Macromolecules, 1995, 28, 991-1002.

[20] Belsito, S.; Bartucci, R; Sportelli, L. Lipid chain length effect on the phase behaviour of PCs/PEG:2000-PEs mixtures. A spin label electron spin resonance and spectrophotometric study. Biophys. Chem., 2001, 93, 11-22.

[21] Pick, U. Liposomes with a large trapping capacity prepared by freezing and thawing of sonicated phospholipid mixture. Arch. Biochem. Biophys., 1981, 212, 186-194.

[22] Boni, L.T.; Stewart, T.P.; Alderfer, J.L.; Hui, S.W. Lipidpolyethylene glycol interactions: I. induction of fusion between liposomes. J. Membrane. Biol., 1981, 62, 65-70.

[23] Frohlich, M.; Brecht, V.; Peschka-Suss, R. Parameters influencing the determination of liposome lamellarity by 31P-NMR. Chem. Phys. Lipids., 2001, 109, 103-12.

(c) Sriwongsitanont and Ueno; Licensee Bentham Open.

This is an open access article licensed under the terms of the Creative Commons Attribution Non-Commercial License (http://creativecommons.org/licenses/by-nc/3.0/) which permits unrestricted, non-commercial use, distribution and reproduction in any medium, provided the work is properly cited. 